\title{
SERIES REARRANGEMENTS AND ANALYTIC SETS
}

\author{
BY
}

\author{
G. G. LORENTZ and K. ZELLER \\ Wayne State Universily, Detroit (Michigan) ${ }^{1}$ )
}

1. Introduction. The rearrangement set of a series $\sum u_{m}$ is the set of the sums of all convergent rearrangements of this series. If instead of ordinary convergence some other convergence concept, $A$-summability, is used in this definition, we obtain the $A$-rearrangement set of the series. Thus, the well-known theorem of Riemann expresses the fact that the rearrangement set of a series of real terms is either empty, or consists of one point, or is the real line. Steinitz characterized rearrangement sets of series of complex terms. In a paper overlooked by reviewing journals, Mazur [6] found $C_{1}$-rearrangement sets of the form

$$
\{\alpha+\beta j\}, \quad j=0, \pm 1, \pm 2, \ldots,
$$

and Bagemihl and Erdös [1] proved that all $C_{1}$-rearrangement sets of real series are either of the form (1) or are given by Riemann's theorem (see section 17 for further details and remarks).

In the present paper we characterize rearrangement sets obtained by arbitrary matrix methods $A$ (in series-to-series form or sequence-to-sequence form, see section 2 and section 16). We restrict ourselves to series and matrices with real terms, which makes simpler some considerations and quotations. The results, however, can be extended to series and matrices with complex terms (or even terms of more general nature), as mentioned in the remarks to our theorems. Every $A$-rearrangement set is an analytic set (Theorem 1; for the definition of analytic sets see section 3 ). The proof depends on a general theorem on ranges of Baire functions. Conversely, every analytic set is the $A$-rearrangement set of a certain series for a regular method $A$

(1) This work was supported by the National Science Foundation grants G 1975 and G 3468 held by the authors at Wayne State University.

11 - 583802. Acta mathematica. 100. Imprimé le 31 décembre 1958. 
(Theorem 2). The construction of $A$ is quite laborious, ideas underlying it are ex plained in section 5 . In the last sections we indicate some further results and problems connected with rearrangement sets.

\section{Rearrangement sets are analytic sets}

2. Summability mothods. In I and II we consider methods $A$ based on matrix transforms $A$ applied to series. For our rearrangement problems, methods of this type are easier to handle than methods based on transforms of sequences, which we treat in section 16. The $A$-summability of a series $u=\sum u_{m}$ to the value $\sigma$ means that the numbers

$$
v_{n}=\sum_{m=1}^{\infty} a_{n m} u_{m} \quad(n=1,2, \ldots)
$$

exist and form a convergent series $v=\sum v_{n}$ with sum $\sigma$. This series is called the $A$-transform of $u$ and denoted $v=A u$. A method $A$ is called regular if it sums each convergent series to its ordinary sum. It is easy to see that the following simple conditions ensure the regularity of $A$ :

$$
\begin{aligned}
& \sum_{n=1}^{\infty} a_{n m}=1 \quad(m=1,2, \ldots), \\
& \sum_{m=1}^{\infty} \sum_{n \neq n_{m}}\left|a_{n m}\right|<\infty,
\end{aligned}
$$

where $n_{m}$ is a sequence of integers depending on $A$. We shall apply this criterion in the following way. For an arbitrary matrix $C$ we put

$$
\|C\|=\sum_{n=1}^{\infty} \sum_{m=1}^{\infty}\left|c_{n m}\right| .
$$

If now a matrix $B$ satisfies $\|I-B\|<\infty$ (where $I$ is the identity matrix) and all column sums $\sum_{n} b_{n m}$ of $B$ are equal to one, then $B$ defines a regular method of type (2). The regularity still remains if we alter $B$ by inserting into $B$ as new rows all the rows of a matrix $D$ for which $\|D\|<\infty$ and all column sums are equal to zero. For further use in section 9 we remark that the norm defined in (5) has the property

$$
\|B C\| \leqslant\|B\|\|C\|
$$

and that the matrix transform $t=C s$ (if it exists) satisfies

$$
\sup \left|t_{l}\right| \leqslant\|C\| \sup \left|s_{k}\right| \text {. }
$$


3. Analytic sets. We give the definition of analytic sets (or Suslin sets) and describe some of their properties (compare the books of Hahn [2], Hausdorff [3], Kuratowski [4]). Given a system $\mathcal{S}$ of subsets $S$ of an arbitrary space we get the system $\mathfrak{S}^{A}$ of analytic sets generated by $\mathfrak{S}$ in the following way: We form a "Suslin scheme",

$$
\left\{S_{k_{1}}, S_{k_{2} k_{2}}, \ldots, S_{k_{1} k_{2} \ldots k_{p}}, \ldots\right\}
$$

with the sets $S$ taken from $\mathfrak{S}$. In the scheme the indices $k_{j}$ run through all positive integers. The sets with $p$ subscripts, i.e. the sets $S_{k_{1}, \ldots, k_{p}}$ are said to be in the $p$ th layer of the scheme. The scheme defines a set $T$ by

$$
T=\bigcup_{\left(k_{1}, k_{\mathrm{s}}, \ldots\right)} \bigcap_{p=1}^{\infty} S_{k_{1}, \ldots k_{p}}
$$

where the union is taken over all possible sequences $k_{1}, k_{2}, \ldots$. Hence in every intersection in (9) exactly one set from each layer appears, however not in an arbitrary way: The sets taken from the $p$ th and the $(p+1)$ st layer must be related in the sense that their first $p$ subscripts are identical. (We can say that the sets of the scheme form a tree.) The system $\subseteq^{A}$ consists of all sets $T$ which can be obtained in this way. One shows easily that $\left(\Im^{A}\right)^{A}=\Im^{A}([2$, p. 344]; $[3$, p. 92]).

Analytic sets of a topological space are by definition the analytic sets generated by the system of open sets. Every Borel set is analytic; but generally the converse does not hold. If the space is the real line (with the usual topology) then all analytic sets are already generated by the system of closed intervals. This follows from the relation $\left(\mathfrak{S}^{A}\right)^{A}=\mathfrak{S}^{A}$, if one takes into account that all open sets are among the analytic sets generated by closed intervals. Similarly we see that an analytic set on the real line which does not contain the point 0 is generated by closed intervals not containing this point. In what follows, analytic sets will always mean analytic sets of the real line (or, in some remarks, of the complex plane).

4. Rearrangement sets are analytic sets. We state the first theorem announced in the introduction.

THEOREM 1. If $A$ is an arbitrary real matrix method of type (2) and $\sum u_{m}$ is an arbitrary series with real terms, then the A-rearrangement set of $\sum u_{m}$ is an analytic set.

Remark. The method $A$ is not necessarily regular. The theorem can be extended to matrices and series with complex terms (or even terms of a more general nature), 
if we use at the end of the proof Theorem XIII in [3, p. 269] instead of Theorem IX.

Proof. Let $N$ be the set whose points $x=\left\{m_{k}\right\}$ are sequences of distinct positive integers. For $x=\left\{m_{k}\right\}, y=\left\{n_{k}\right\}$ we define

$$
\varrho(x, y)=\frac{1}{l} \quad \text { if } m_{k}=n_{k} \quad \text { for } k<l \text { and } m_{l} \neq n_{l} .
$$

This makes $N$ a metric space and one sees easily that $N$ is complete and separable.

We show that $N$, the set of $x \in N$ such that the $m_{k}$ take all positive integral values, is a Borel subset of $N$. Let

$$
N\left(a_{1}, \ldots, a_{l-1} ; p\right)
$$

be the set of $x$ with $m_{k}=a_{k}(k<l)$ and $m_{l}=p$. Then

$$
N_{p}=\bigcup_{l=1}^{\infty} \bigcup_{a_{1}} N\left(a_{l-1}, \ldots, a_{l-1} ; p\right)
$$

is the set of $x$ such that $m_{k}=p$ for some $k$. Finally

$$
N=\bigcap_{p=1}^{\infty} N_{p}
$$

Since the set (11) is an open ball in $N$, the set $\bar{N}$ is a Borel subset of the space $N$.

Now for the method $A$ and the series $\sum u_{m}$ in question we put for each $x \in N$

$$
f_{n l}(x)=\sum_{k=1}^{l} a_{n k} u_{m_{k}} .
$$

Clearly $f_{n l}$ is a continuous function on $N$. Hence (see for example [3, p. 270]), the set $C_{n}$ of the points $x$ of the existence of the limit

$$
f_{n}(x)=\lim _{l \rightarrow \infty} f_{n l}(x)
$$

is a Borel set of $N$. Again, $f_{n}(x)$ are Baire functions in the metric space $C=\cap C_{n}$ (which is a Borel subset of $N$ ). As Baire functions the $f_{n}$ have the property that the sets of points $x$ where $f_{n}(x)>\alpha$ or $\geqslant \alpha$ (for a fixed $n$ ) are Borel sets [3, p. 260]. Therefore the set $C^{*}$ of the points $x$ of the existence of

$$
f(x)=\sum_{n=1}^{\infty} f_{n}(x)
$$


is a Borel subset of $C$ (see [3, p. 272]) and hence a Borel subset of $N$. The Baire function $f$, defined in the metric space $C^{*}$, maps the set $C^{*} \cap N$ exactly into the $A$-rearrangement set $R$ of $\sum u_{m}$. Now $C^{*} \cap N$ is a Borel set and hence also an analytic set in the metric space $C^{*}$. But $C^{*} \cap \bar{N}$ is also a Borel set and hence an analytic set in the complete space $N$. Therefore $C^{*} \cap N$ is an absolutely analytic set in the sense of Hausdorff $\left[3\right.$, p. 121 and p. 208]. Since $C^{*} \cap \bar{N}$ is separable, we can apply $[3$, p. 266, Theorem IX] and obtain that $R$ is analytic. This completes the proof.

\section{Analytic sets are rearrangement sets}

5. Statement of the theorem and outline of the proof. In our considerations we use the following series

$$
e^{1 !}+0+e^{2 !}+0+e^{3 !}+\cdots
$$

At a later place (section 15) we indicate a rather general class of series containing (16) for which all our arguments remain valid.

THEOREM 2. Given any analytic set $T$ of the real line, there exists a regular matrix method of type (2) such that $T$ is the A-rearrangement set of the series (16).

Outline of the proof. We sketch the main ideas of our construction by considering first the case where $T$ is a finite closed interval, denoting the matrix as in the actual proof by $A$. Let $q=\sum q_{m}$ be the subseries of (16) consisting of all non-zero terms. We break $q$ up into countably many subseries $q^{(j)}=\sum q_{m}^{(j)}$. The matrix $\bar{A}$ will have the property that in an $A$-summable rearrangement of (16) each $q^{(i)}$ can appear essentially on two places (each "place" is an infinite sequence of columns of $\bar{A}$ ). At one place it causes an $A$-sum $\varrho_{2 j-1}$, at the other the sum $\varrho_{2}$. The matrix $A$ is so constructed that the sums caused by different $q^{(j)}$ are just added. Hence the $A$-sum $\varrho$ of a rearrangement of (16) is of the form $\sum \sigma_{j}$, where each $\sigma_{j}$ is either $\varrho_{2 j-1}$ or $\varrho_{2} j$.

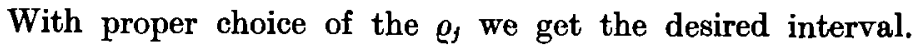

We now explain why each $q^{(j)}$ has essentially only two places to go. There are certain matrices $\bar{B}$ used in the construction of $A$ which are coarse, i.e. have the property that they sum essentially only one (or certain specified subseries) of (16) (see section 8 ). The reason that we can work rather independently with the $q^{(1)}$ is due to the fact that $A$ is constructed from matrices $\overline{A_{3}}$ in a special way called mixing (see section 6): the columns of $A$ are the columns of all $\bar{A}_{j}$, diluted by zeros, and similarly for rows. For some special series $y$ connected with (16), $A$-summability is 
equivalent to the assumption that for every $j$ the subseries of $y$ "belonging" to $\bar{A}_{j}$ is summed by the latter method.

The full proof of the theorem will consist of three steps, which we distinguish by the sets $T$ which can be obtained at each step: one point sets (matrices are denoted by $\bar{A}, \ldots$; section 9 ), intervals (matrices $A, \ldots$; section 10 ), general analytic sets (matrices $A, \ldots$; section 13). To construct the matrices $A$ at each step we define matrices $B, C, D, E$; their rows will be combined to form $A$. In the case of an interval, $\bar{B}$ has the task to restrict the possible rearrangements and $\bar{C}$ will provide the proper $A$-sums, while the matrices $\bar{D}$ and $\bar{E}$ are used only for the last step (analytic sets): $\bar{D}$ provides additional relations for subseries of (16) summable by $A$; and $\bar{E}$ is responsible for connections between the sums assigned to such subseries by the matrices $A_{j}$ used in the construction of $A$, connections which are similar to those appearing in the Suslin scheme. Finally we have to remark that on the first two steps (one point sets and intervals) we have to work with rather general subseries of (16) rather than with (16) itself.

In sections 6-8 we first introduce notations: mixing, special series, coarse matrices. Sections 9-13 are then devoted to the actual construction of the matrix $A$.

6. Mixing of series and matrices. Let $u=\sum u_{m}$ be a series; by $N$ we denote the set of all positive integers. Let $P=\left\{p_{1}, p_{2}, \ldots\right\}$ be an infinite subset of $N$ (where $\left.p_{1}<p_{2}<\ldots\right)$. The projection $u^{P}$ of $u$ onto $P$ will be the series

$$
u^{P}=\sum_{m \in P} u_{m}=u_{p_{1}}+u_{p_{1}}+\ldots
$$

In other words, this is a series obtained by omission from $u$ of all terms for which $m$ does not belong to $P$; the remaining terms retain their relative order. Conversely, let $u^{*}=\sum u_{m}^{*}$ be a series. Its injection onto $P$ is the series $u=\sum u_{m}$ such that

$$
u_{p_{k}}=u_{k}^{*} \quad(k=1,2, \ldots), u_{m}=0 \text { otherwise. }
$$

We shall also consider the corresponding notions for matrices. The projection of a matrix $A$ onto $P$ is the matrix $A_{P}$ with the elements

$$
a_{n m}^{P}=a_{p_{n} p_{m}} \quad(n, m=1,2, \ldots) .
$$

The injection of a matrix $A^{*}$ onto $P$ is the matrix $A$ with elements

$$
a_{p_{l} p_{k}}=a_{l k}^{*} \quad(l, k=1,2, \ldots), a_{n m}=0 \text { otherwise }
$$


Next we define mixtures of series and matrices. In the following considerations we always use a fixed partition of $N$ into disjoint sets $P_{j}$ :

$$
N=P_{1} \cup P_{2} \cup \ldots ; \quad P_{1}=\left\{p_{j 1}, p_{j 2}, \ldots\right\}
$$

For example, we can take

$$
P_{1}=\{1,3,5,7, \ldots\}, \quad P_{2}=\{2,6,10,14, \ldots\}, \quad P_{3}=\{4,12,20,28, \ldots\}, \ldots
$$

The mixture of the series $u^{(j)}=\sum u_{m}^{(j)}(j=1,2, \ldots)$ is the series $u=\mathfrak{M}\left(u^{(1)}, u^{(2)}, \ldots\right)$ with the terms

$$
u_{m}=u_{k}^{(j)} \quad \text { if } m=p_{j k}
$$

In other words: $u$ is the termwise sum of the injections of the $u^{(f)}$ onto $P_{j}$. Similarly we define the mixture of matrices $A_{j} \quad(j=1,2, \ldots)$ to be the matrix $A=\mathfrak{M}\left(A_{1}, A_{2}, \ldots\right)$ with the elements

$$
a_{n m}=a_{l k}^{(j)} \quad \text { if } n=p_{l l}, m=p_{j k} ; \quad a_{n m}=0 \text { otherwise. }
$$

Again $A$ is the termwise sum of the injections of the $A_{j}$ onto $P_{j}$, and the projection of $A$ onto $P_{j}$ is $A_{j}$. For an arbitrary matrix $A$ (not necessarily of the type (24)) we often write for the projection $A_{P_{j}^{\prime}}$ shorter $A_{j}$; similarly for series, $u^{(j)}$ instead of $u^{P}$.

As a simple example of the result of mixing we mention

$$
\mathfrak{M}(I, I, \ldots)=I \text {. }
$$

Further we have $\quad\left\|\mathfrak{M}\left(A_{1}, A_{2}, \ldots\right)\right\|=\left\|A_{1}\right\|+\left\|A_{2}\right\|+\ldots$.

It follows that if $B_{f}=I-B_{j}^{*}$, then

$$
\mathfrak{M}\left(B_{1}, B_{2}, \ldots\right)=I-B^{*}, \quad \text { where }\left\|B^{*}\right\|=\sum\left\|B_{f}^{*}\right\| \text {. }
$$

In particular, if the $B_{f}$ define regular summability methods (see section 2) and $\sum\left\|B_{j}^{*}\right\|<\infty$, then also $\mathfrak{M}\left(B_{1}, B_{2}, \ldots\right)$ is a regular method.

Suppose that $A$ is the mixture of matrices $A_{f}$, and that $u^{\mathrm{P}_{f}}=u^{(j)}$ are the projections of a series $u$. Then the transform $v=A u$ consists of all the terms of the transforms $v^{(j)}=A_{j} u^{(j)}$. If now $u$ is $A$-summable, then necessarily for every $j$ the series $v^{(j)}$ has terms tending to zero, and only finitely many series $v^{(i)}$ contain large terms:

$$
\lim _{n} v_{n}^{(j)}=0 \quad(j=1,2, \ldots) ; \quad \sup _{n}\left|v_{n}^{(j)}\right|<1 \quad\left(j>j_{0}\right)
$$


On the other hand, if $\quad \sum_{j} \sum_{n}\left|v_{n}^{(j)}\right|<\infty$,

then $u$ is $A$-summable.

We also use modifications of the mixing process. The matrix $A$ is called the column-mixture of the matrices $A_{j}(j=1,2, \ldots)$, if the columns of $A$ are the unaltered columns of the $A_{j}$ arranged in a way prescribed by the sets $P_{j}$ :

$$
a_{n m}=a_{n k}^{(j)} \quad \text { if } m=p_{j k} \text {. }
$$

It amounts to the same to say that the $n$th row of $A$, considered as a series, is the mixture of the $n$th rows of the $A_{1}$ (considered as series). Similarly we define rowmixtures of matrices $A_{j}(j=1,2, \ldots)$. The row mixture of matrices $A_{1}, \ldots, A_{r}, 0,0, \ldots$ is also called the row-mixture of $A_{1}, \ldots, A_{r}$. (For our purposes we could as well omit the zero rows and define the row-mixture of $A_{1}, \ldots, A_{r}$ as a matrix $A$ which is formed by the rows of the $A_{1}, \ldots, A_{r}$ in such a way that every row of each $A_{1}, \ldots, A_{r}$ appears in $A$ exactly once.)

7. Special series. It will be convenient to introduce special notations for subseries of our fundamental series (see also (16))

$$
e^{1 !}+0+e^{2 !}+0+e^{3 !}+\cdots
$$

We shall denote by

$$
y=\sum_{m=1}^{\infty} y_{m}
$$

any rearrangement of a subseries of (31); in section 13, however, $y$ shall mean a rearrangement of (31) itself. By $q=\sum q_{m}$ we denote special subseries with terms

$$
q_{m}=e^{k_{m} !} \quad\left(0<k_{1}<k_{2}<\cdots\right)
$$

or, in section 13, with terms $\dot{q}_{m}=e^{m !}$.

Two series $u=\sum u_{m}$ and $u^{\prime}=\sum u_{m}^{\prime}$ will be called equivalent, if

$$
u_{m}=u_{m}^{\prime} \text { for almost all } m \text {. }
$$

About series $y, q$ of types (32), (33) we note the following.

LEM A 1. If $y_{n}-\lambda q_{n}(n=1,2, \ldots)$ is bounded for some constant $\lambda$, then $\lambda=0$ or $\lambda=1$ and $y_{n}-\lambda q_{n}=0$ for all large $n$; and if $\left|y_{n}-\lambda q_{n}\right| \leqslant 2,(n=1,2, \ldots)$, then $y_{n}-\lambda q_{n}=0$ for all $n$.

For the proof we note that $\lambda=\lim \left(y_{n} / q_{n}\right)$, and this can only be 0 or 1 . Then $y_{n}-\lambda q_{n}$ is $y_{n}$ or $y_{n}-q_{n}$, and these expressions are bounded only if they vanish for 
all large $n$. The last part of the lemma follows from the fact that the difference of any two terms of $(31)$ is either 0 or greater than 2 in absolute value.

8. Coarse matrices. For each matrix $B$ we consider the set $B^{l}$ of all series of type (32) orthogonal to $B$, i.e. with the property that the $B$-transform exists and is identically zero. Hence

$$
u \in B^{\perp} \text { means: } u \text { is of type (32) and } B u=0
$$

(where 0 stands for the series $\sum 0$ ). Note that $B^{\perp}$ by definition contains only series of form (32).

A matrix $B$ is called coarse (with respect to the series (31)) if it has the following properties:

If a series $y$ (of type (32)) has a transform $z=B y$ with $\sup \left|z_{n}\right|<\infty$, then it is $B$-summable and equivalent to a series from $B^{\perp}$. If it satisfies $\sup \left|z_{n}\right|<1$, then it is in $B^{1}$.

L вм м 2. The series $y=\sum y_{m}$ (of type (32)) summable by a mixture $B$ of regular, coarse matrices $B$, are exactly the series $y$ which are equivalent to a series belonging to $B^{\perp}$.

Proof. By $z^{(j)}$ we denote the $B_{j}$-transform of the projection $y^{(j)}$ of $y$ onto $P_{j}$. If $y$ is $B$-summable, we have by (28)

$$
\sup _{n}\left|z_{n}^{(j)}\right|<\infty \quad(j=1,2, \ldots) ; \quad \sup _{n}\left|z_{n}^{(j)}\right|<1 \quad\left(j>j_{0}\right) .
$$

From the definition (36) it follows then that $y$ is equivalent to a series in $B^{\perp}$. The converse of this is trivial, since by the regularity assumption all columns is $B$ form convergent series.

9. One-point sets. Basic for our construction are the matrices of the following type

$$
\bar{B}=\left[\begin{array}{cccccccrc}
\mathrm{I} & 0 & 0 & \ldots & 0 & \beta_{2} & 0 & 0 & \ldots \\
0 & 1 & 0 & \ldots & 0 & \beta_{3} & 0 & 0 & \ldots \\
0 & 0 & 1 & \ldots & 0 & \beta_{4} & 0 & 0 & \ldots \\
. & . & . & \ldots & . & \dot{\beta}_{k} & . & . & \ldots \\
0 & 0 & 0 & \ldots & \mathbf{1} & 0 & 0 & \ldots \\
0 & 0 & 0 & \ldots & 0 & 1-\beta_{2}-\ldots-\beta_{k} & \beta_{k+1} & 0 & \ldots \\
0 & 0 & 0 & \ldots & 0 & 0 & 1-\beta_{k+1} & \beta_{k+2} & \ldots \\
. & . & . & \ldots & . & . & . & . & \ldots
\end{array}\right]
$$

Their main properties are described in the following lemma. 
LEMMA 3. For each series $q=\sum q_{m}$ (of type (33)) and each $\varepsilon>0$ there exists $a$ coarse matrix $\bar{B}$ such that

$$
\|I-\overline{\bar{B}}\|<\varepsilon
$$

and that

$$
\overline{B^{\perp}} \text { consists of the series } q \text { and } 0 \text {. }
$$

Proof. We select the coefficients $\beta_{m}$ inductively in such a way that $q$ is orthogonal to $\vec{B}$, in other words in such a way that

$$
\left.\begin{array}{rl}
q_{m}+\beta_{m+1} q_{k}=0 & (m=1, \ldots, k-1), \\
\left(1-\beta_{2}-\cdots-\beta_{k}\right) q_{k}+\beta_{k+1} q_{k+1}=0, & \\
\left(1-\beta_{m}\right) q_{m}+\beta_{m+1} q_{m+1}=0 & (m=k+1, k+2, \ldots) .
\end{array}\right\}
$$

Then each series $u$ with $\dot{B} u=0$ is of the form

$$
\left.u=\lambda q \quad \text { (i.e. } u_{m}=\lambda q_{m}, m=1,2, \ldots\right) \text {. }
$$

In case $u$ is of type (32) we have $\lambda=0$ or $\lambda=1$ by Lemma 1 . Hence (40) is true. By taking $k$ sufficiently large we can achieve (39).

It remains to prove that $\bar{B}$ is coarse. We may assume $\varepsilon<\frac{1}{2}$. The matrix $\dot{B}$ has an inverse

and (compare (6))

$$
\bar{B}^{-1}=I+(I-\bar{B})+(I-\overline{\bar{B}})^{2}+\cdots
$$

$$
\left\|\dot{B}^{-1}\right\| \leqslant 1+\varepsilon+\varepsilon^{2}+\cdots=\frac{1}{1-\varepsilon}<2 .
$$

If now a series $y$ (of type (32)) is such that its $\bar{B}$-transform $z=\ddot{\bar{B}} y$ satisfies $\sup \left|z_{n}\right|<\infty$, then it has a decomposition

$$
y_{m}=w_{m}+u_{m} \quad \text { with } \quad w=\bar{B}^{-1} z \quad \text { and } \bar{B} u=0 .
$$

Hence by (41) (see also (7)) and the same consideration as in (42) we have

$$
y_{m}=w_{m}+\lambda q_{m} \quad \text { with } \sup \left|w_{m}\right|<\infty
$$

Applying Lemma 1 we see that $w_{m}=0$ for $m \geqslant m_{0}$ and the first part of (36) is proved. The second part follows similarly, observing that $\sup \left|z_{n}\right|<1$ implies $\sup \left|w_{m}\right|<2$ in (46).

The matrix $B$ sums $q$ to 0 . It is very easy to make this sum an arbitrary number $\varrho$ by inserting some additional rows into $\overline{\bar{B}}$. Put 


$$
\bar{C}=\left[\begin{array}{rrrrrrrrrrrrrr}
0 & \ldots & 0 & \gamma_{1} & 0 & \ldots & . & . & . & \ldots & . & . & . & \ldots \\
0 & \ldots & 0 & -\gamma_{1} & 0 & \ldots & 0 & \gamma_{2} & 0 & \ldots & . & . & . & \ldots \\
0 & \ldots & 0 & 0 & 0 & \ldots & 0 & -\gamma_{2} & 0 & \ldots & 0 & \gamma_{3} & 0 & \ldots \\
. & \ldots & . & . & . & \ldots & . & . & . & \ldots & . & . & . & \ldots
\end{array}\right]
$$

We define the $\gamma_{k}$ by the condition that the series $r=\overline{\bar{C}} q$ satisfies

$$
r_{1}=\varrho, r_{n}=0(n>1)
$$

Taking the non-zero columns in $\check{C}$ sufficiently far to the right we can achieve

$$
\|\bar{C}\|<\varepsilon
$$

Our remark about the value of the sum of $q$ may be then justified as follows. Let $\bar{A}$ be the row-mixture of $\bar{B}$ and $\bar{C}$ (see section 6 ). The matrix method $\bar{A}$ is regular (see (3), (4); note that the column sums of $\bar{B}$ are $I$ and those of $\bar{C}$ are zero). It sums among the series $y$ (of type (32)) exactly those equivalent to $q$ or to 0 . Because $\overline{\bar{A}}$ sums $q$ to the value $\varrho$ and is regular, the $\overline{\bar{A}}$-rearrangement set of $q$ consists exactly of the point $\varrho$. It is easy to modify $\bar{A}$ so that the summed series is (31) instead of $q$; this provides a proof of Theorem 2 in the simple case of a one-point set. We omit the details because this case will be covered by our more general construction.

10. Intervals. Let

$$
[\alpha, \beta], \quad-\infty<\alpha<\beta<\infty
$$

be a closed interval. We consider a fixed series $q$ (of type (33)) and a number $\varepsilon>0$. The projections $q^{(j)}$ of $q$ onto $P_{1}$ are again series of type (33). For each $j=1,2, \ldots$ we select a real number $\varrho_{f}$, for the moment arbitrary, and a number $\varepsilon_{f}>0$ such that $2 \sum \varepsilon_{f}<\varepsilon$. The matrices $\bar{B}_{21-1}=\bar{B}_{2 j}$ will denote the matrix $\bar{B}$ of section 9 corresponding to the series $q^{(j)}$ and the bound $\varepsilon_{j}$ in (39). The matrices $\bar{C}_{2 j-1}, \bar{C}_{2}$, are the matrices $\bar{C}$ of section 9 corresponding to the series $q^{(j)}$, the bound $\varepsilon_{j}$ in (49) and the sum values $\varrho_{2 j-1}$ and $\varrho_{2}$, respectively. Let

$$
\bar{B}=\mathfrak{M}\left(\bar{B}_{1}, \bar{B}_{2}, \ldots\right), \quad \bar{C}=\mathfrak{M}\left(\bar{C}_{1}, \bar{C}_{2}, \ldots\right)
$$

be the mixtures of these matrices as described in section 6 . The matrix $\bar{B}$ has the property stated in Lemma 2 . In addition we can easily describe the set $\bar{B}^{1}$. This set consists of all series $y$ (of type (32)) such that the projections $y^{(j)}$ onto $P$, for each $j=1,2, \ldots$ satisfy 


$$
\left.\begin{array}{lll}
\text { either } & y^{(2 j-1)}=q^{(j)} & \text { and } y^{(2 f)}=0 \\
\text { or } & y^{(2 j-1)}=0 \quad \text { and } y^{(2 f)}=q^{(j)} \\
\text { or } & y^{(2 j-1)}=y^{(2 f)}=0 .
\end{array}\right\}
$$

We now specify the numbers $\varrho_{\text {, by putting }}$

$$
\varrho_{1}=\varrho_{2}=\alpha ; \quad \varrho_{2 j-1}=2^{-j+1}(\beta-\alpha) \quad \varrho_{2 j}=0 \quad(j>1) .
$$

The $\overline{\bar{C}}_{2 j-1}$-transform of the series $q^{(j)}$ is the series $\varrho_{2 j-1}+0+0+\cdots$, while its $\overline{\bar{C}}_{2 j^{-}}$ transform is $\varrho_{2},+0+0+\cdots$. The $\bar{C}$-transform of a $y \in \bar{B}^{1}$ is the mixture of these series; and the resulting (mixed) series is convergent because $\sum\left|\varrho_{3}\right|<\infty$. The possible sum-values of the $\bar{C}$-transform are

$$
\varrho=\sum \sigma_{j}, \quad \text { where } \sigma_{j}=\varrho_{2 j-1} \text { or } 0 .
$$

They form the union of the two intervals $[\alpha, \beta]$ and $[0, \beta-\alpha]$. If however $y \in \bar{B} \perp$ is a rearrangement of

$$
q_{1}+0+q_{2}+0+q_{3}+\cdots,
$$

then the last case mentioned in (52) cannot happen, and the possible sum-values give exactly the interval $[\alpha, \beta]$.

We are now in the position to prove Theorem 2 in the case where the set $T$ is an interval. Again we omit details because this case is covered by our general construction in section 13. Let $\bar{A}^{\prime}$ be the row-mixture of $\bar{B}$ and $\bar{C}$ (see section 6). The summability method $\bar{A}^{\prime}$ is regular by (3), (4) and (26). Among the rearrangements of the series (55) this method will sum by Lemma 2 exactly those equivalent to a series in $\bar{B}^{\perp}$. We can therefore (by the regularity of $\bar{A}^{\prime}$ ) restrict ourselves to the consideration of series in $\bar{B}^{\perp}$. From the preceding paragraph it follows that the interval $[\alpha, \beta]$ is the rearrangement set of the series (55) with respect to $A^{\prime}$. With a trivial choice of the $q_{m}$ the series $(55)$ will be identical to (31), hence the special case of the theorem is proved.

11. The auxiliary matrices $D$. The matrix $A^{\prime}$ just defined has the following disadvantage for the further construction: It sums certain series $y \in \bar{B}^{\perp}$ which are neither 0 nor a rearrangement of $q$ (namely series where the last case mentioned in (52) happens for some but not all $j$ ). To prevent this we insert further rows into the matrix $A^{\prime}$.

Given a series $q=\sum g_{m}$ (of type (33)) we can determine a matrix (compare $\ddot{C}$ in (47)) 


$$
\bar{D}=\left[\begin{array}{rrrrrrrrrr}
0 & \ldots & 0 & \delta_{1} & 0 & \ldots & . & . & . & \ldots \\
0 & \ldots & 0 & -\delta_{1} & 0 & \ldots & . & . & . & \ldots \\
0 & \ldots & 0 & 0 & 0 & \ldots & 0 & \delta_{2} & 0 & \ldots \\
. & \ldots & . & . & . & \ldots & 0 & -\delta_{2} & 0 & \ldots
\end{array}\right]
$$

such that $r=\bar{D} q$ is the series $1-1+1-1+\cdots$ :

$$
\ddot{D} q=\sum(-1)^{m+1}
$$

and that

$$
\|\overline{\bar{D}}\|<\varepsilon \text {. }
$$

Let $\overline{\bar{D}}_{1}=\bar{D}_{2}$ be matrices of type (56) corresponding to the series $q^{(1)}$, where $q^{(j)}$ has the same meaning as in section 10; and let $\bar{D}_{3}=\overline{\bar{D}}_{4}$ be matrices of this type for the series $q^{(2)}$. The column-mixture $\bar{D}^{*}$ of the matrices $\ddot{\bar{D}}_{1}, \bar{D}_{2},-\overline{\bar{D}}_{3},-\overline{\bar{D}}_{4}, 0,0, \ldots$ has the property that for each $y \in \bar{B}^{\perp}$

$$
\begin{aligned}
& z=\bar{D}^{*} y=0 \text { holds if either } y^{(j)}=0 \quad(j=1, \ldots, 4) \\
& \text { or the last case in (52) does not happen for } j=1,2 ; \\
& \text { otherwise } \overline{\lim }\left|z_{n}\right|=1 .
\end{aligned}
$$

(We see that for instance in case $y^{(1)}=q^{(1)}, y^{(3)}=q^{(3)}$ the contributions from $\overline{\bar{D}}_{1}$ and $\bar{D}_{3}$ cancel out.)

Similarly we get matrices which have the corresponding property for the quadruple $(3,4,5,6)$ instead of $(1,2,3,4)$, and so on. The row-mixture $\bar{D}$ (see section 6 ) of all these matrices $\bar{D}^{*}$ has the property that for each $y \in \bar{B}^{\perp}$

$$
\begin{aligned}
& \begin{aligned}
z=\bar{D} y=0 \quad \text { holds if either } y=0 \\
\quad \text { or the last case in (52) does not happen for any } j \text {; }
\end{aligned} \\
& \text { otherwise } \varlimsup\left|z_{n}\right|=1 .
\end{aligned}
$$

We can furthermore achieve $\|\bar{D}\|<\varepsilon$.

We now form the row-mixture $A$ of the matrices $A^{\prime}$ and $\bar{D}$. Besides the properties mentioned in section 10 for $A^{\prime}$ (regularity, rearrangement set) the new $A$ will also satisfy

$$
\begin{aligned}
& \text { If } y \in \bar{B}^{\perp} \text { and } z_{n} \rightarrow 0 \quad \text { (for } z=A y \text { ), } \\
& \text { then either } y=0 \\
& \text { or } y \text { is a rearrangement of the series (55). }
\end{aligned}
$$


12. The auxiliary matrices $\boldsymbol{E}$. The matrix $\bar{D}$ serves to restrict the projections of $\bar{A}$-summable series. For the construction in section 13 based on a Suslin scheme we need further matrices $E$ providing relations among the sums which the projections $\bar{A}_{j}$ of the matrix $A$ assign to the projections $u^{(j)}$ of a series $u$. For a given series $q$ (of type (33)) and a number $\varepsilon>0$ we form the matrix

$$
\overline{\bar{E}}=\left[\begin{array}{rrrrrrrrrr}
0 & \ldots & 0 & \gamma_{1} & 0 & \ldots & . & . & . & \ldots \\
0 & \ldots & 0 & -\gamma_{1} & 0 & \ldots & . & . & . & \ldots \\
0 & \ldots & . & . & . & \ldots & 0 & \gamma_{2} & 0 & \ldots \\
0 & \ldots & . & . & . & \ldots & 0 & -\gamma_{2} & 0 & \ldots \\
. & \ldots & . & . & . & \ldots & . & . & . & \ldots
\end{array}\right]
$$

where the $\gamma_{i}$ and their column numbers are the same as in $\overline{\bar{C}}$ (see (47)). Hence $\overline{\bar{E}}$ transforms $q$ into the series

$$
\varrho-\varrho+\varrho-\varrho+\cdots
$$

where $\varrho$ is the sum assigned to $q$ by $\overline{\bar{A}}$.

Based on $\overline{\bar{E}}$ we construct a matrix $\bar{E}$ which has an analogous property for intervals instead of one-point sets. Let $\overline{\bar{E}}_{2 j-1}, \overline{\bar{E}}_{2}$, be matrices (63) corresponding to the series $q^{(j)}$ and the sum-values $\varrho_{2 j-1}, \varrho_{2 j}$ respectively (where $q^{(j)}$ and $\varrho_{j}$ have the same meaning as in section 10). The column-mixture of the matrices $\overline{\bar{E}}_{j}$ (see section 6 ) is row-infinite and has the property:

If $y \in \bar{B}^{\perp}$ is $\bar{C}$-summable to the value $\varrho$,

then the $\bar{E}$-transform of $y$ is the series $\varrho-\varrho+\varrho-\varrho+\cdots$.

Further, all column-sums in $\bar{E}$ are zero and we can achieve

$$
\| \bar{E} \mid<\varepsilon
$$

Using the $\bar{E}$ in a similar way as the $\overline{\bar{D}}$ in section 11 we shall enforce that certain sums of the type mentioned in the first paragraph of this section are equal (see section 13).

13. Analytic sets. We represent an arbitrary analytic set $T$ by a Suslin scheme of closed intervals:

$$
T=\bigcup_{\left(k_{p}, k_{g}, \cdots,\right.} \bigcap_{p=1}^{\infty} S_{k_{1}, \cdots, k_{p}}
$$


In case $0 \notin T$ we may assume that none of the intervals $S_{k_{1}, \ldots, k_{p}}$ contains 0 (see section 3). To abbreviate writing, we consider a fixed one-to-one correspondence

$$
j \leftrightarrow\left(k_{1}, \ldots, k_{p}\right)
$$

between the set of all subscripts $\left(k_{1}, \ldots, k_{p}\right)$ and the set of all positive integers $j$; we shall use the index $j$ instead of $\left(k_{1}, \ldots, k_{p}\right)$, and say that this $j$ belongs to the level $p$.

By $y$ we denote a rearrangement of the series (31) and by $q$ the subseries of positive terms of (31) (given by $k_{m}=m$ in (33)).

We decompose $q$ into countably many subseries $q^{(p)}$. Each $q^{(p)}$ will be assigned to the $p$-th layer (see section 3) of the scheme (67). For each $j \leftrightarrow\left(k_{1}, \ldots, k_{p}\right)$ we define according to sections 10-12 matrices $\bar{B}_{j}, \bar{C}_{j}, \bar{D}_{j}, \bar{E}_{j}, A_{j}$ corresponding to the series $q^{(p)}$, the intervals $S_{f}$ and the numbers $\varepsilon_{j}>0$ with

$$
\sum \varepsilon_{j}<\infty
$$

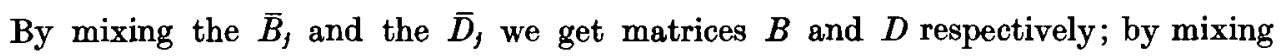
the matrices

$$
\bar{C}_{f}^{\prime}=\left\{\begin{array}{lr}
\bar{C}_{f} & \text { if } j \leftrightarrow\left(k_{1}\right), \\
0 & \text { otherwise }
\end{array}\right.
$$

we get a matrix $C$. Let $j^{*} \leftrightarrow\left(k_{1}^{*}, \ldots, k_{p}^{*}\right)$ be an arbitrary subscript. We put

$$
\bar{E}_{f}^{\prime}=\left\{\begin{array}{cc}
\bar{E}_{j} & \text { if } j \leftrightarrow\left(k_{1}^{*}, \ldots, k_{p}^{*}\right), \\
-\bar{E}_{j} & \text { if } j \leftrightarrow\left(k_{1}^{*}, \ldots, k_{p}^{*}, k\right), \\
0 & \text { otherwise. }
\end{array}\right.
$$

The column-mixture of the matrices $\bar{E}_{j}^{\prime}=E_{j}^{\prime}\left(j^{*}\right)$ is called $E_{j *}$. We can arrange $\|E\|<,\varepsilon_{y}$, and then

$$
\sum\left\|E_{i}\right\| \leqslant \sum \varepsilon_{j}<\infty
$$

The main property of the matrices $E$, is the following. Let

$$
j \leftrightarrow\left(k_{1}, \ldots, k_{p}\right), \quad j^{\prime} \leftrightarrow\left(k_{1}, \ldots, k_{p}, k\right) .
$$

We consider a rearrangement $y$ of the series (31) which belongs to $B^{\perp}$. If $y$ is $E_{y^{-}}$ summable, then the $C_{y}$-sum of the projection $y^{(j)}$ of $y$ is equal to the sum (over $k$ )

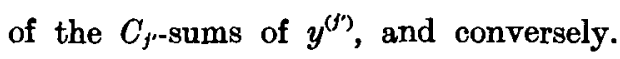


We define the matrix $E$ to be the row-mixture (see section 6 ) of the $E$. Let $\sigma_{j}=\sigma_{j}(y)$ denote the $C_{j}$-sum (if it exists) of the projection $y^{(j)}$ of a series $y$. We have then:

$$
\begin{aligned}
& \text { A series } y \in B^{\perp} \text { is } E \text {-summable if and only if } \\
& \text { for arbitrary } j, j^{\prime} \text { connected by (73), } \sigma_{j}=\sum_{k} \sigma_{j^{\circ}} \text {. }
\end{aligned}
$$

Now we define the matrix $A$ as the row-mixture of $B, C, D, E$. The matrix $A$ determines a regular summability method (2) since it satisfies (3), (4) because of the conditions imposed on the column-sums and norms of the matrices from which $A$ is constructed. We have to show that $T$ is the $A$-rearrangement set of the series (31).

Assume first that a rearrangement $y$ of the series (31) is $A$-summable to $\varrho$. We wish to prove that $\varrho \in T$. As a mixture of the $\bar{B}_{j}$ the matrix $B$ is also a mixture of matrices $\bar{B}$ and has therefore the property stated in Lemma 2. Hence we can restrict our considerations to the case $y \in B^{\perp}$. By $(62)$ we see that the projections $y^{(j)}, j \leftrightarrow\left(k_{1}, \ldots, k_{p}\right)$ of $y$ have the property

$$
\text { Either } y^{(j)}=0 \text { or } y^{(j)} \text { is a rearrangement of } q^{(p)} \text {. }
$$

Since the subseries $q^{(p)}$ are disjoint, we see that for each level $p$ there is exactly one $j=j_{p}$ of this level with

$$
y^{(j)}=q^{(p)} \quad \text { for } j=j_{p}, \quad y^{(j)}=0 \text { for all other } j \text { of this level. }
$$

Because the $A$-sum $\varrho$ of $y$ is determined only by the $\bar{C}$, with $j \leftrightarrow\left(k_{1}\right)$, we see that

$$
\varrho \in \bigcup_{k_{1}=1}^{\infty} S_{k_{i}}
$$

Finally the matrix $E$ is responsible for the fact that even $\varrho \in T$. If $j_{1} \leftrightarrow\left(k_{1}^{*}\right)$, we see from (76) that $\varrho=\sigma_{1}$ and $\varrho \in S_{k_{i}^{*}}$. We now distinguish the cases $\varrho \neq 0$ and $\varrho=0$. First let $\varrho \neq 0$. Since $y^{\left(f_{1}\right)}$ is $\bar{C}_{f_{1}}$-summable to the value $\varrho=\sigma_{f_{1}}, y^{(j)}$ is $\bar{C}_{f}$-summable to 0 for all $j \neq j_{2}$ of the second level, and $y^{\left(j_{s}\right)}$ is $\bar{C}_{j_{2}}$-summable to $\sigma_{j_{3}}$, we see from (74) that $j_{2}$ must be of the form $j_{2} \leftrightarrow\left(k_{1}^{*}, k_{2}^{*}\right)$, and that $\sigma_{j_{a}}=\varrho \neq 0$. Hence by the properties of $\bar{C}_{j_{1}}, \varrho \in S_{k_{i}^{*} k_{i}^{*}}$. Continuing we find

$$
\varrho \in \bigcap_{p=1}^{\infty} S_{k_{i}^{*} \ldots \ldots k_{p}^{*}} \subset T .
$$

Next let $\varrho=0$. From $\varrho \in S_{k_{i}^{*}}$ we deduce that $0 \in S_{k_{1}}^{*}$ and hence $0 \in T$ (because we agreed to take intervals $S$ with $0 \notin S$ if $0 \notin T)$. 
A short argument follows to show that if $\tau \in T$, then there is a rearrangement $y$ summable to $\tau$. Since $\tau \in T$, for proper subscripts $k_{1}^{*}, k_{2}^{*}, \ldots$ we have

$$
\tau \in \bigcap_{p=1}^{\infty} S_{k_{i}^{*} \ldots . . k_{p}^{*}}
$$

We choose now a rearrangement $y$ of the series (31) such that the projections $y^{(j)}$ satisfy

$$
\left.\begin{array}{l}
y^{(j)} \quad \text { is a rearrangement of } q^{(p)} \text { if } j \leftrightarrow\left(k_{1}^{*}, \ldots, k_{p}^{*}\right), \\
y^{(j)}=0 \text { otherwise. }
\end{array}\right\}
$$

By section 10 and the definition of the $\bar{C}$, we can select the rearrangement $y^{(f)}$ in such a way that

$$
\bar{C}_{f}\left(y^{(j)}\right)=\tau+0+0+\cdots .
$$

By (70), $y$ is $C$-summable to $\tau$; by (81), (74) and (60) we see that the $E$ - and $D$ transforms of $y$ are identically zero. We also have $y \in B^{\perp}$; hence $y$ is $A$-summable to $\tau$. This completes the proof of Theorem 2 .

Remark. Theorem 2 remains true for analytic sets $T$ of the complex plane. We have only to select the $\varrho_{i}$ in section 10 so as to obtain a complex (two-dimensional) interval $S$ instead of a real interval.

\section{Additional remarks}

14. Modifications of the construction. The construction leading to the matrix $A$ of Theorem 2 can be modified in several ways. We mention two possibilities.

The auxiliary matrix $D$ served the following purpose. If a rearrangement $y$ of

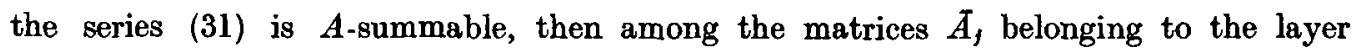
$p$ one gets essentially the full series $q^{(p)}$, while zeros appear in the columns reserved for the other $A_{j}$, of this level (see (76)). The same result can be achieved in a different way without the use of an auxiliary matrix. The breaking up of series $q^{(p)}$ into subseries (section 10) is modified: for each $p$ and each $j \leftrightarrow\left(k_{1}, \ldots, k_{p}\right)$ we break up $q^{(p)}$ into subseries $q^{(p)}(j, k), k=1,2, \ldots$, in a manner essentially different for each $j$ of a level $p$. This means that for $j \neq \bar{j}$, the series $q^{(p)}(j, k)$ and $q^{(p)}(\bar{j}, k)$ have infinitely many terms in common.

The second modification avoids the exceptional role played by the point 0 in the proof of Theorem 2 at the cost of introducing another auxiliary matrix, similar to $E$, which - regardless of the sums involved - enforces a connection between the places where the non-zero terms of an $A$-summable rearrangement of (31) may appear. 
15. Extensions of the construction. As we have mentioned in section 5, more general series than (16) can be used in Theorem 2. Examining the proof of this theorem (see in particular Lemma 1) we see that it more generally applies to a series

$$
u_{1}+0+u_{2}+0+u_{3}+\cdots
$$

with the properties

$$
0<u_{1} \leqslant u_{2} \leqslant \cdots ; \quad u_{n+1} / u_{n} \rightarrow \infty ;
$$

without loss of generality we can then assume $u_{1}>2, u_{n+1}-u_{n}>2,(n=1,2, \ldots)$. We cannot work, however, with an arbitrary divergent series. If the method $A$ is regular, then for some divergent series $u=\sum u_{m}$ we easily recognize certain restrictions of the possible $A$-rearrangement sets of $u$ (compare section 17).

The constructed matrix $A$ is rather pathological. Already the matrices $B$ have rather peculiar properties (Einfolgenverfahren, see [7]).

Perhaps it is possible to replace the $D_{j}$ in the construction of $A$ by matrices of the type

$$
\boldsymbol{F}=\left[\begin{array}{cccccc}
\frac{1}{2} & 0 & 0 & 0 & 0 & \ldots \\
\frac{1}{2} & \frac{1}{2} & 0 & 0 & 0 & \ldots \\
0 & \frac{1}{2} & \frac{1}{6} & 0 & 0 & \ldots \\
0 & 0 & \frac{5}{6} & \frac{5}{8} & 0 & \ldots \\
0 & 0 & 0 & \frac{1}{6} & \frac{1}{24} & \ldots \\
\cdot & . & . & . & . & \ldots
\end{array}\right]
$$

The matrix $F$ has quite acceptable properties from the general point of view (perfectness, see [7]) and is also related to known special methods (weighted arithmetic means, Zweierverfahren [7]). It sums the series $2 !-2 !+3 !-3 !+\cdots$ and the $F$ rearrangement set consists exactly of the two points 1 and -1 . Matrices like $F$ make one expect that even in case of "smooth" methods like Abel's or Borel's rather complicated rearrangement sets are possible.

16. Sequence transformations. The summability methods of Theorem 2 have been based on transforms (2) of series $\sum u_{m}$ into series $\sum v_{n}$. It is natural to consider transforms of the sequence $s_{k}=u_{1}+\cdots+u_{k}$ of the partial sums of $\sum u_{m}$ into a series $\sum v_{n}$ :

$$
v_{n}=\sum_{k=1}^{\infty} a_{n k}^{*} s_{k} \quad(k=1,2, \ldots)
$$

which also define summability methods. From (85) we easily derive an equivalent transform of the $s_{k}$ into the $t_{l}=v_{1}+\cdots+v_{l}$. However, the equivalence problem of 
(2) and (85) is not trivial. One would expect that with $a_{n k}^{*}=a_{n k}-a_{n, k+1},(2)$ and (85) define the same summability method. However, this is not generally true if $A$ is row-infinite, since the equation

$$
\sum_{m=1}^{\infty} a_{n m} u_{m}=\sum_{k=1}^{\infty} a_{n k}^{*} s_{k}
$$

does not always hold (compare [5]). Nevertheless, from Theorem 2 we can derive:

There is a sequence-to-series (or a sequence-to-sequence) method (85) which has the properties described by Theorem 2.

For the proof we consider the validity of (86). This relation is certainly true if $A$ is row-finite, and more generally if the series $\sum a_{n m} u_{m}$ has only finitely many non-vanishing terms for each $A$-summable $u$. The matrices $B, C, D$ of Theorem 2 are row-finite; we shall show that a slight change of the matrix $E$ achieves our requirement.

For each $A$-summable rearrangement $y$ of (31) we see from (76) that in the $E_{j *}$ transform of $u$ actually appear (see (71)) only at most two of the infinitely many

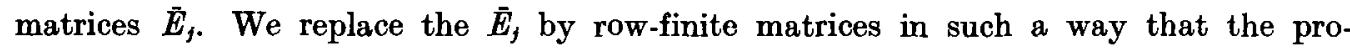
perty (74) is preserved.

Replacing some elements of each row by zeros, we obtain for each $\delta>0$ from the matrix $\bar{E}$ of section 12 a row-finite matrix $\bar{F}$ which instead of (65) has the property:

If $y \in \vec{B}^{\perp}$ is $\bar{C}$-summable to $\varrho$, then the $\bar{F}$-transform of $y$ is a series

$$
\tau_{1}-\tau_{1}+\tau_{2}-\tau_{2}+\cdots \text { with }\left|\tau_{m}-\varrho\right|<2^{-m} \delta \text { (so that } \tau_{m} \rightarrow \varrho \text { ). }
$$

Replacing in (71) the $\bar{E}_{j}$ by $\bar{F}_{j}$ corresponding to $\delta_{j}>0$ with $\sum \delta_{j}<\infty$, we obtain instead of $E$ a matrix $F$ such that a series $y \in B^{\perp}$ is $E$-summable if and only if it is $F$-summable, and that (74) is still true. The row-mixture of $B, C, D, F$ defines then a method $A$ with the property that $A^{*}$ contains $A$ if we restrict ourselves to rearrangements of the series (31).

On the other hand, this construction can be so arranged that in each row of $A$, non-zero elements are separated by zeros. Then we have $A^{*} \subset A$ for arbitrary series. This follows from the fact that the series $\sum a_{n k}^{*} s_{k}$ is obtained from $\sum a_{n m} u_{m}$ simply by replacing each term by two, namely by $-a_{n m} s_{m-1}$ and $a_{n m} s_{m}$. Hence the convergence of the second series is implied by that of the first. This completes the proof.

Actually even more is true. We can choose the matrix $A$ in Theorem 2 to be row-finite. The proof requires some computations and estimates, and we outline it 
only. Using the matrices $F$ described above, we can construct (modified) $F_{j}$ in such a way that under the circumstances described in section 13 , the transform for the series which we want to exclude is $\eta_{1}-\eta_{1}+\eta_{2}-\eta_{2}+\cdots$, where $\eta_{k} \rightarrow \alpha_{1} \varrho$ with some $\alpha_{j} \neq 0$, while for the series which we want to preserve, $\eta_{n}$ converges rapidly to zero and satisfies $\left|\eta_{n}\right| \leqslant \alpha_{j} \varrho$. If we insert the rows of the (modified) matrices $F_{j}$ into $A$ in such a way that also in the $A$-transform $\eta_{n}$ and $-\eta_{n}$ are consecutive terms, if we further choose the $\alpha_{j}$ small enough, then the matrix $F$ will destroy $A$-summability of exactly the "undesirable" series, so that the set $T$ will again be the $A$-rearrangement set of (31), but now with a row-finite matrix $A$.

17. The papers of Mazur and Bagemihl-Erdös. The proofs of Mazur [6] and Bagemihl-Erdös [1] of the existence of $C_{1}$-rearrangement sets of type (1) depend on a gap theorem for $C_{1}$. The method can be used to show that for most summability methods, $A$-rearrangement sets of type (1) appear.

Mazur also showed that for series $\sum u_{m}$ with bounded real terms the $C_{1^{-}}$and the Abel-rearrangement sets are the same as in Riemann's theorem. The two main steps in Mazur's proof are: Treatment of special series $\gamma+\delta+\gamma+\delta+\cdots$; dilution of the series $\sum u_{m}$ to $u_{1}+0+u_{2}+0+\cdots$ (which does not change $C_{1}$-summability). Instead of this the $A K$-property of $C_{1}$ (see [7]) could be used. For general regular methods $A$ one can only prove that the $A$-rearrangement set of the series $\gamma+\delta+\gamma+\delta+\cdots$ (and of similar series) contains with a point $\varrho$ also the points $\varrho+k(\gamma-\delta)(k= \pm 1$, $\pm 2, \ldots)$.

The most difficult part of the proof of the theorem of Bagemihl and Erdös, the characterization of all $C_{1}$-rearrangement sets, depends on the estimate $s_{k}=o(k)$ which is valid for a $C_{1}$-summable sequence $s_{k}$. This ensures small changes of the $C_{1}$-transform if the series is rearranged cautiously. This estimate is connected with the property of $C_{1}$ known as the "mean value theorem" or "Abschnittskonvergenz" [7]. For matrices with this and some additional smoothness properties the considerations of Bagemihl and Erdös can still be carried through. Unfortunately such matrices define rather weak summability methods. The question remains open what happens in the case of stronger methods like $C_{\alpha}(\alpha>1)$, Abel and Borel methods. 


\section{References}

[1]. F. Bagemul \& P. ERdös, Rearrangements of $C_{1}$-summable series. Acta Math., 92 (1954), 35-53.

[2]. H. HarN, Reelle Funktionen, 1. Teil. Leipzig, 1932.

[3]. F. HAUSDORFF, Mengenlehre. Berlin, 1935.

[4]. C. Kuratowskr, Topologie, vol. 1. Warszawa, 1952.

[5]. G. G. LoRENTz, Über Limitierungsverfahren, die von einem Stieltjes-Integral abhängen. Acta Math., 79 (1947), 256-273.

[6]. S. MazUR, Über bedingt summierbare Reihen (Polish with German summary). Arch. Tow. Nauk. Lwow. Wydz. III, Mat.-Przyr. IV (1929), 411-424.

[7]. K. ZELLeR, Theorie der Limitierungsverfahren (Ergebnisse der Mathematik). Berlin 1958. 